\title{
Club des Laboratoires Accrédités : partage d'expérience, audits croisés et amélioration de la qualité des mesures
}

\author{
Nicholas BOUILLON, David BENHAMOU, Laure DOMENECH, Boris GEYNET et Loïc ROSO \\ CT2M, Centre des creusets, Route de Lançon, 13250 SAINT CHAMAS, France CT M.
}

\begin{abstract}
Résumé. Depuis 2011, le Club de Laboratoires Accrédités se réunit une fois par semestre et le nombre de participants est chaque fois grandissant. Les premières réunions ont regroupé une trentaine de participants venant de laboratoires aussi variés qu'EDF, CEA Cadarache, LDA 13, PROTEC LEA, LASEM Marine nationale, Institut Coopératif du Vin, LERM, SCP, Avepharm, IGNA, Quadlab, ITGA, EUROCOPTER,... Ces laboratoires sont tous accrédités ou en démarche d'accréditation selon l'ISO 17025.La participation au Club des Laboratoires Accrédités est totalement gratuite. Les réunions se font sur une demi-journée dans un laboratoire accueillant qui est différent à chaque fois, et se déroulent en quatre temps : Sans un premier temps : présentation des évolutions de l'accréditation ISO 17025 à travers un thème d'actualité. Dans un deuxième temps, il y a des Echanges entre les laboratoires accrédités sur les relations avec les auditeurs Cofrac et sur les écarts récurrents (à travers la présentation d'écarts d'audits Cofrac des laboratoires participants). Dans un troisième temps, il y a des échanges d'auditeurs et présentation des pages internet du Club des Laboratoires Accrédités dédiées aux audits croisés. Et enfin dans un quatrième et dernier temps, il y a des visites du laboratoire accueillant et plus particulièrement des activités accréditées.
\end{abstract}

\section{Présentation générale du club des laboratoires accrédités}

Le CT2M est un lieu privilégié d'échange d'informations et d'expériences pour les entreprises et les laboratoires.

Depuis l'année 2011 le CT2M organise et anime le Club des Laboratoires accrédités qui se réunit 2 fois par an un sur des thèmes chaque fois différents.

Ces réunions regroupent chaque fois une trentaine de participants venant de laboratoires de taille et de domaine d'activité très variés tel qu'EDF, CEA Cadarache, LDA 13, PROTEC LEA, LASEM Marine nationale, Institut Coopératif du Vin, LERM, SCP, Avepharm, IGNA, Quadlab, ITGA, EUROCOPTER,...

L'ensemble de ces laboratoires sont tous accrédités ou en démarche d'accréditation selon l'ISO 17025, certains d'entre eux sont aussi certifiés ISO 9001 et 14001 ce qui permet d'avoir une grande diversité en terme d'approche en qualité et ainsi d'enrichir la qualité des débat et des échanges entre les laboratoires.

La participation au Club des Laboratoires Accrédités est totalement gratuite grâce à la participation de la
Région Provence Alpes Côte d'Azur et de l'Europe via des fond Feder.

Les réunions se font sur une demi-journée dans un laboratoire accueillant qui est différent à chaque fois, à la fois au niveau de son domaine d'activité afin de varier les expériences et les «cultures » des laboratoires, que d'un point de vue géographique afin de pas privilégier de laboratoires d'une « région » plus qu'un autre.

\section{Le déroulement des réunions du club des laboratoires accrédités :}

Les réunions se déroulent en quatre temps : Sans un premier temps : présentation des évolutions de l'accréditation ISO 17025 à travers un thème d'actualité.

Dans un deuxième temps, il y a des échanges entre les laboratoires accrédités sur les relations avec les auditeurs Cofrac et sur les écarts récurrents (à travers la présentation d'écarts d'audits Cofrac des laboratoires participants).

Dans un troisième temps, il y est fait un point sur le programme d'échanges d'auditeurs internes ( audits croisés). 
Et enfin dans un quatrième et dernier temps, il y a une visite du laboratoire accueillant en orientant la visite plus particulièrement sur les activités accréditées COFRAC.

\subsection{Présentation des évolutions de l'accréditation ISO 17025 à travers un thème d'actualité :}

\subsection{1 - Première réunion du club des laboratoires accrédités sur l'approche processus}

La première réunion $\mathrm{du}$ club des laboratoires accrédités s'est tenu à Aix, au laboratoire du Canal de Provence, le vendredi 2 décembre 2011.

A cette occasion l'évolution du référentiel des laboratoires vers une approche processus a été abordé.

Dans un premier temps, il a été rappelé ce qu'est un processus en repartant de la définition tirée de l'ISO 9000 , ensuite il a été présenté les 3 principaux type de processus :

- les processus de réalisation,

- les processus support

- les processus pilote ou de management.

Une fois ces définitions abordées, l'objectif a été de voir comment mettre en place l'approche processus en 3 grandes étapes :

- identification du cœur d'activité du laboratoire et ses missions,

- identification et classement des différents processus

- réalisation de la cartographie du laboratoire

Dans la mise en place de cette approche, il a été souligné l'importance du choix des pilotes de processus ainsi que de la définition de bon indicateurs.

Enfin les avantages et inconvénients de cette approche ont été débattus

\subsection{2 - Deuxième réunion du club des laboratoires accrédités sur les Essais Interlaboratoires}

La deuxième réunion $\mathrm{du}$ club des laboratoires accrédités s'est tenu au CT2M le vendredi 13 avril 2012 matin

Ce petit déjeuner a permis notamment de faire un point sur le thème des Essais inter-laboratoires : en abordant une première question fondamentale "Comment exploiter ses essais inter-laboratoires ?.

Pour cela il a été précisé les 3 principaux objectifs poursuivis lors de l'organisation d'un Essai Interlaboratoire :

- attribuer une valeur consensuelle à une caractéristique d'un objet,

- estimer l'exactitude d'une méthode

- évaluer les performances des laboratoires.
Il a été ensuite rappelé l'importance pour le COFRAC à promouvoir ces comparaisons interlaboratoires et aux laboratoires accrédités, l'obligation de participer à ces essais lorsqu'ils existent.

Les résultats de ces essais sont en effet examinés par les auditeurs lors des audits et les laboratoires doivent prendre des mesures particulières lorsque les résultats aux EIL sont en dehors des limites.

Les limites que les laboratoires se fixent ont été abordées notamment au niveau du calcul du $\mathrm{Z}$ score ou de l'écart normalisé.

Hormis cette exploitation « obligatoire » des essais inter laboratoire, d'autres exploitations ont été abordées acomme de suivre la performance du laboratoire dans le temps et de détecter des tendances non visibles en observant chaque $\mathrm{Z}$ score séparément.

Une autre exploitation des EIL a été traitée afin d'estimer les incertitudes d'essais suivant la méthode ISO 5725-2 et la méthode SH GTA 14.

Cette réunion du club des laboratoires accrédités a permis aussi d'abordant une deuxième question fondamentale "Comment faire, lorsqu'il n'existe pas d'essais inter-laboratoires, pour répondre aux exigences du Cofrac?".

Plusieurs pistes ont été abordées comme l'organisation d'une simple comparaison avec un autre laboratoire, de recourir à l'utilisation régulière de matériaux de référence ou de réitérer ses essais ou étalonnages à l'aide de méthodes équivalentes.

Enfin hormis l'exigence de l'ISO 17025, le groupe a mis en évidence, l'intérêt que tout laboratoire a de participer à ces EIL car c'est un outil de progrès important, il est utile pour la comparaison de méthodes, il permet d'assurer la qualité des résultats délivrés par le laboratoire et de démontrer la compétence du laboratoire.

\subsection{3 - Troisième réunion $d u$ club des laboratoires accrédités sur les portées flexibles}

La troisième réunion du club des laboratoires accrédités s'est tenu au laboratoire d'EDF CEIDRE TEGG, à Aix en Provence, le vendredi 30 novembre 2012 matin.

Le thème développé lors de cette réunion concernait la gestion de la portée flexible et ISO 17025 : "Qu'est-ce qu'une portée flexible ? Qui est concerné par la rédaction d'une procédure de gestion de la portée flexible ? Que doit-on mettre dans une telle procédure ?".

Dans un premier temps, il a été rappelé ce qu'est une portée d'accréditation en repartant de la définition du COFRAC et en présentant différents exemples de portées. 
Ensuite il a été présenté les différences entre une portée fixe (type A1) et une portée flexible et les différents niveaux de flexibilité ont été détaillés (type A2, A3 et B).

Les intérêts pour le laboratoire en fonction des différents niveaux de flexibilité ont été largement abordés aussi bien au niveau de sa capacité à pouvoir répondre rapidement à une évolution des besoins d'un client que sur l'aspect important du coût pour le laboratoire.

Une douzaine de questions très pratiques relatives aux portées flexibles ont ensuite été traitées comme «Une accréditation en portée flexible coûte-t-elle plus cher qu'une accréditation en portée fixe ? », « Comment les clients du laboratoire peuvent-ils savoir que la portée du laboratoire est flexible ? » ou encore « Un laboratoire non encore accrédité peut-il directement demander l'accréditation en portée flexible? » «Avec quel niveau de détail faut-il exprimer sa portée flexible (champs de possibilités) ?» « Une portée d'accréditation peut-elle être mixte (champs fixes et champs flexibles) ?» .

Pour finir une exemple de gestion de portée flexible a été présenté de manière simple et complète sous la forme d'un diagramme.

\section{1 .4 - Quatrième réunion du club des laboratoires accrédités sur la dématérialisation des rapports}

La quatrième réunion du club des laboratoires accrédités s'est tenu à l'APAVE, à Châteauneuf les Martigues, le vendredi 14 juin 2013.

Le thème développé lors de cette réunion traitait de la dématérialisation des rapports : Quelles sont les exigences de Cofrac ? Comment faire en pratique dans son laboratoire?.

Dans un premier temps, il a été évoqué les différentes niveaux où la dématérialisation des données peut intervenir dans un laboratoire :

- conservation des documents du SMQ,

- conservation des données brutes des essais et traitement informatique,

- production du rapport,

- $\quad$ approbation du rapport,

- $\quad$ transmission du rapport

- $\quad$ archivage du rapport.

Il a été rappelé aux participants que le COFRAC a mis à la disposition des laboratoires un Guide Technique d'Accréditation à ce sujet, le LAB GTA 09 « dématérialisation des données dans les laboratoires.

Dans un deuxième temps, les exigences concernant la dématérialisation des données dans le cadre de la génération des rapports ont été revues.

La gestion dématérialisée des rapports et leur transmission doivent suivre les mêmes exigences que les rapports papier, à savoir : l'intégrité des données, l'identification de l'approbateur et enfin la confidentialité des données.

$\mathrm{Au}$ sujet de l'approbation électronique des rapports, plusieurs solutions sont possibles et peuvent être mise en œuvre :

- $\quad$ signature électronique présumée fiable,

- LIMS où la signature est apposée par reconnaissance par le système d'un couple identifiant / mot de passe, numérisation du document papier,

- apposition d'un fichier « image » dans un document de type « word » avant conversion au format pdf.

Chaque solution d'approbation électronique a été détaillée au cours de la réunion.

Un autre aspect de la dématérialisation concerne aussi la transmission des rapports qui peut être faite : par courriel, sur support (clé USB, CD, DVD, ...), par mise à disposition sur un site dédié ou par liaison informatique dédiée.

Enfin les précautions à prendre lors de la revue de contrat ont été précisées car elles sont particulières et fondamentales.

En effet L'émission d'un document autre qu'un document signé manuscritement et envoyé par courrier nécessite l'établissement d'une convention de preuve.

Cette convention de preuve permet de définir les modalités :d'établissement (document papier / numérique), de signature (électronique, via LIMS, ...), de transmission (mails, extranet, support numérique, ...) et d'archivage des documents (format et durée).

Enfin pour finir 4 exemples d'écarts rencontrés lors des audits COFRAC ont été cités et discutés.

\subsection{Echanges entre les laboratoires accrédités sur les relations avec les auditeurs Cofrac et sur les écarts récurrents}

La réunion se poursuit avec des échanges entre les laboratoires accrédités sur les relations qu'ils ont avec les auditeurs Cofrac ainsi que sur les écarts récurrents.

On demande à ce que les laboratoires participants présentent un ou plusieurs écarts d'audits Cofrac aux autres laboratoires participants et ces écarts sont discutés et commentés ensemble.

\subsection{Echanges d'auditeurs internes}

Le club des laboratoires accrédité est aussi une « bourse d'échange » d'audit internes.

En effet, les laboratoires accrédités ont l'obligation de réaliser un audit interne de leur laboratoire une fois par an. Il est intéressant pour un laboratoire de faire appel 
régulièrement ou de temps à autre, à un auditeur d'un autre laboratoire afin d'avoir un regard extérieur.

Dans le but de diminuer le coût de cet audit, le CT2M propose de favoriser les échanges d'audits internes. Pour cela le CT2M a développé sur son site des pages internet dédiées au Club des Laboratoires Accrédités pour les audits croisés.

Les laboratoires voulant y participer peuvent envoyer au CT2M leur CV qui sera mis sur le site afin de créer un « pool»d'auditeurs compétents.

Puis les laboratoires intéressés par cette formule pourront, soit consulter les $\mathrm{CV}$ puis rentrer directement en relation avec l'auditeur de son choix, soit faire une demande d'audit au CT2M qui sera relayée via le site.

\section{4 visites du laboratoire accueillant}

Les réunions du club des laboratoires accrédité se terminent par une visite du laboratoire accueillant, en orientant la visite plus particulièrement sur les activités accréditées COFRAC

Ceci a amené les participants à découvrir :

- le tout nouveau Pôle laboratoires du Canal de Provence,

- le laboratoire d'étalonnage du CT2M,

- le laboratoire d'EDF CEIDRE TEGG

- le laboratoire de l'Apave et de la cellule métrologie

\section{Conclusion}

Ces rencontres entre laboratoires accrédités connaissent un vif succès.

Elles sont en effet très enrichissantes pour l'ensemble des participants car elles leurs permettent de s'informer et d'échanger sur des thèmes d'actualité et de partager leurs expériences ou difficultés, de manière pratique et aussi conviviale.

La bourse d'échange d'audit proposé aux laboratoires du club est aussi un moyen simple et peu onéreux pour répondre à l'exigence d'audit interne pour un bon nombre d'entre eux.

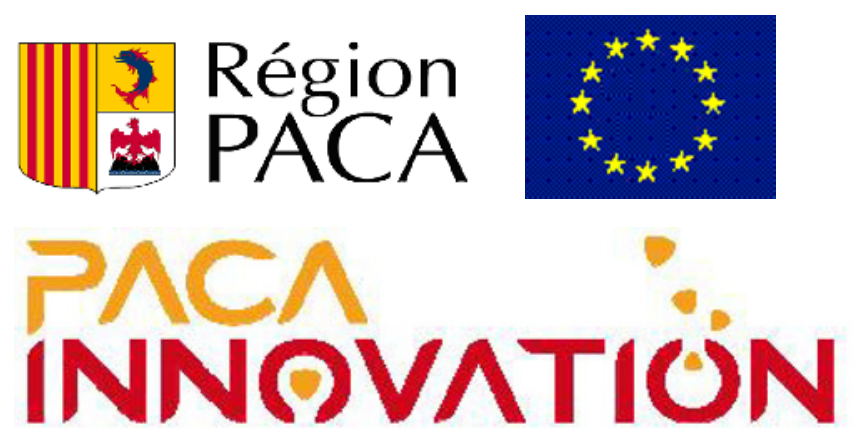

